\title{
Recursion Operators and Frobenius Manifolds ${ }^{\star}$
}

Franco MAGRI

Dipartimento di Matematica ed Applicazioni, Università degli Studi di di Milano Bicocca, Via Roberto Cozzi 53, 20125 Milano, Italy

E-mail: franco.magri@unimib.it

Received June 01, 2012, in final form October 05, 2012; Published online October 19, 2012

http://dx.doi.org/10.3842/SIGMA.2012.076

\begin{abstract}
In this note I exhibit a "discrete homotopy" which joins the category of Fmanifolds to the category of Poisson-Nijenhuis manifolds, passing through the category of Frobenius manifolds.
\end{abstract}

Key words: F-manifolds; Frobenius manifolds; Poisson-Nijenhuis manifolds

2010 Mathematics Subject Classification: 35D45; 53D17; 37K10

\section{Introduction}

In this short note I wish to present some ideas of a recent comparative study of three different types of manifolds which are of interest in different areas of physics and mathematics. They are:

1) F-manifolds;

2) Frobenius manifolds;

3) bi-Hamiltonian manifolds;

The study is addressed to emphasize a point of view allowing to see these three different kinds of manifolds under a unified perspective, almost as three different instances (or realizations) of a single geometrical idea.

Let us start by recalling first the definitions of the manifolds we are interested in.

F-manifolds. F-manifolds have been introduced by Hertling and Manin in 1999 [1]. They are manifolds where it is allowed to multiply vector fields according to a rule of the form:

$$
\partial_{j} \circ \partial_{k}=C_{j k}^{l} \partial_{l}
$$

The multiplication is assumed to be commutative, associative and with unity. The structure constant $C_{j k}^{l}$ are the components of a symmetric vector-valued 2-form $C: T M \times T M \rightarrow T M$, and the unity is a special vector field $e: M \rightarrow T M$. According to Hertling and Manin the manifold $M$ is a F-manifold if the multiplication $C$ and the unity $e$ verify the conditions [2]:

1) $\operatorname{Lie}_{e}(C)=0$;

2) $\operatorname{Lie}_{X \circ Y}(C)=X \circ \operatorname{Lie}_{Y}(C)+\operatorname{Lie}_{X}(C) \circ Y$.

Frobenius manifolds. Frobenius manifolds are manifolds where each tangent space has the structure of a Frobenius algebra. The notion of Frobenius manifold has been introduced by Boris Dubrovin in 1992 [3]. According to him, a manifold equipped with a multiplication $C: T M \times T M \rightarrow T M$, with a unity $e: M \rightarrow T M$, and with a metric $g: T M \times T M \rightarrow \mathbb{R}$ (not necessarily positive definite) is a Frobenius manifolds if:

\footnotetext{
*This paper is a contribution to the Special Issue "Geometrical Methods in Mathematical Physics". The full collection is available at http://www.emis.de/journals/SIGMA/GMMP2012.html
} 
1) the metric is flat: $\operatorname{Riemann}(g)=0$;

2) the unity is covariantly constant: $\nabla e=0$;

3) the multiplication is symmetric with respect to the metric: $g(X, Y \circ Z)=g(X \circ Y, Z)$;

4) the covariant differential of the symmetric covariant tensor field $c(X, Y, Z)=g(X, Y \circ Z)$ is symmetric, that is it verifies the symmetry condition: $\nabla_{X} c(Y, Z, W)=\nabla_{Y} c(X, Z, W)$.

It can be shown that the axioms of Dubrovin entail that the multiplication $C$ and the unity $e$ verify the axioms of Hertling and Manin. Therefore, Frobenius manifolds are a particular class of F-manifolds.

Bi-Hamiltonian manifols. Bi-Hamiltonian manifolds have been introduced in 1979. They are manifolds equipped with a pair of compatible Poisson brackets. This means that the pencil

$$
\{f, g\}_{\lambda}=\{f, g\}_{1}+\lambda\{f, g\}_{2}, \quad \lambda \in \mathbb{R}
$$

defined by the pair of Poisson brackets $\{f, g\}_{1}$, and $\{f, g\}_{2}$ satisfies the Jacobi identity for any value of the parameter $\lambda$. If one of the Poisson bracket is symplectic, the bi-Hamiltonian manifold is called a Poisson-Nijenhuis manifold. This type of manifolds has been thoroughly studied in [4].

At first sight, it is difficult to see any point of contact between the definitions of Frobenius manifolds and of Poisson-Nijenhuis manifolds. They belong to different geometries. Frobenius manifolds belong to Riemannian geometry, while Poisson-Nijenhuis manifolds belong to symplectic geometry. Nevertheless it is known, after the work of Witten and Kontsevich, that the physical theories which are behind them, namely the topological field theories and the theory of integrable systems, are closely related. This occurrence leads to suspect that also between the correlated geometrical models there should exist strong points of contact. This idea has motivated the present research.

To attain this unification, I follow a technique of homotopic deformation. I imagine the three different categories of manifolds as three distinct points in an abstract space (the space of all categories of smooth manifolds), and I conceive the idea to search for a simple path connecting these points. The construction of a path which joins the category of F-manifolds to the category of Poisson-Nijenhuis manifolds, passing trough the category of Frobenius manifolds is the main novelty contained in the present paper.

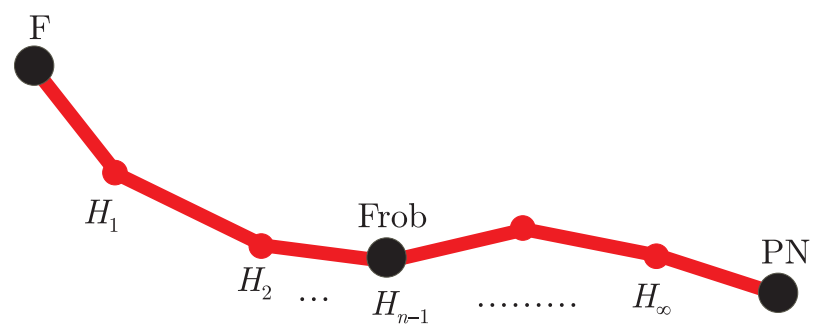

As shown by the figure, this path is actually a polygonal line, whose vertices are denoted by $H_{1}, H_{2}, \ldots, H_{\infty}$. Each vertex is a new category of manifolds, defined according to a regular rule. Moving to the right, towards PN-manifolds, the complexity of the manifolds increases. The manifolds of type $H_{m+1}$ have indeed a richer geometrical structure than the manifolds of type $H_{m}$, in the sense that they are manifolds of type $H_{m}$ endowed with additional geometric proprieties. Therefore the vertices of the polygonal line represent a chain of categories of smooth manifolds obeying the inclusion relations:

$$
H_{1} \supset H_{2} \supset \cdots \supset H_{m} \supset H_{m+1} \supset \cdots \supset H_{\infty}
$$


F-manifolds and PN-manifolds are the terminal points of this chain, since F-manifolds are manifolds of type $H_{1}$ deprived of one structure, while PN-manifolds are manifolds of type $H_{\infty}$ enriched with an additional structure. They are not included into the chain, because their inclusion would destroy the validity of the simple recursive procedure allowing to pass from the category $H_{m}$ to the category $H_{m+1}$. To keep the simplicity of the scheme, they must be considered as limit points not belonging to the chain.

This paper has two specific aims:

1) to explain the definition of the new categories of manifolds $H_{m}$;

2) to show that our polygonal line passes through the category of Frobenius manifolds, by a concrete example.

\section{Manifolds of type $H_{1}$}

The definition of manifolds of type $H_{1}$ comes directly from the theory of bi-Hamiltonian manifolds.

Let $M$ be a bi-Hamiltonian manifold, and let $X$ be a bi-Hamiltonian vector field defined over it. If $P_{1}: T^{*} M \rightarrow T M$ and $P_{2}: T^{*} M \rightarrow T M$ are the Poisson bivectors associated with the pair of Poisson brackets giving $M$ the structure of bi-Hamiltonian manifold, the last requirement means that there exist on $M$ a pair of functions $h_{1}: M \rightarrow \mathbb{R}$ and $h_{2}: M \rightarrow \mathbb{R}$ such that

$$
X=P_{1} \mathrm{~d} h_{1}=P_{2} \mathrm{~d} h_{2} .
$$

Assuming that $P_{1}$ is invertible, let us set

$$
K=P_{2} P_{1}^{-1}
$$

and let us consider the manifold $M$ equipped with the recursion operator $K$, the bi-Hamiltonian vector field $X$, and with the privileged 1 -form $\theta=\mathrm{d} h_{1}$. It is well-known, within the theory of bi-Hamiltonian manifolds, that these three geometric objects satisfy the conditions:

1) $\operatorname{Torsion}(K)=0$

2) $\mathrm{d} \theta=0$

3) $\mathrm{d}(K \theta)=0$;

4) $\operatorname{Lie}_{X}(K)=0$;

5) $\operatorname{Lie}_{X}(\theta)=0$.

To pass from PN-manifolds to the manifolds of type $H_{1}$, one has to modify these conditions in two ways. First, one has to replace the strong condition

$$
\operatorname{Torsion}(K)=0
$$

by the weaker condition

$$
\operatorname{Haantjes}(K)=0 \text {. }
$$

Secondly, one has to balance the weakening of the condition on $K$ by an additional condition on $\theta$. This condition requires that the 1 -form $\theta$ annihilates the torsion of $K$, which is now different from zero. So, the new condition is

$$
\theta(\operatorname{Torsion}(K))=0 \text {. }
$$


Definition 1. A manifold $M$ equipped with a privileged vector field $X: M \rightarrow T M$, with a privileged 1-form $\theta: M \rightarrow T^{*} M$, and with a recursion operator $K: T M \rightarrow T M$ is a manifold of type $H_{1}$ if:

1) $\operatorname{Haantjes}(K)=0$;

2) $\mathrm{d} \theta=0$

3) $\mathrm{d}(K \theta)=0$;

4) $\theta(\operatorname{Torsion}(K))=0$;

5) $\operatorname{Lie}_{X}(K)=0$;

6) $\operatorname{Lie}_{X}(\theta)=0$.

The additional condition, which demands that $\theta$ annihilates the torsion of $K$, has a clear geometrical meaning. Together with the conditions $\mathrm{d} \theta=0$ and $\mathrm{d}(K \theta)=0$, it entails that the second iterated 1-form $K^{2} \theta$ is closed as well. Thus, on any manifold of type $H_{1}$ there is a triple of 1-forms $\left(\theta, K \theta, K^{2} \theta\right)$ which are closed, and therefore locally exact. This triple will be referred to as the short Lenard chain associated with the recursion operator $K$ and the privileged 1-form $\theta$. By denoting by $K_{0}: T M \rightarrow T M$ the identity map on the tangent bundle, and by renaming $K_{1}$ the recursion operator $K$, one may condense the three conditions defining a short Lenard chain in the more compact and neat form

$$
\mathrm{d}\left(K_{j} K_{l} \theta\right)=0, \quad j, l=0,1 .
$$

This equation will be referred to as the definition of the short Lenard chain of a manifolds of type $H_{1}$. Making use of this equation, one may give the following alternative definition of manifolds of type $H_{1}$.

Definition 2. The manifold $M$ is a manifold of type $H_{1}$, if

1) $\operatorname{Haantjes}\left(K_{j}\right)=0$;

2) $\mathrm{d}\left(K_{j} K_{l} \theta\right)=0$

3) $\operatorname{Lie}_{X}\left(K_{j}\right)=0$;

4) $\operatorname{Lie}_{X}(\theta)=0$

for $j, l=0,1$, where $X$ is a privileged vector field and $K_{0}$ the identity map.

To see the relation between manifolds of type $H_{1}$ and F-manifolds, it is enough to concentrate the attention on the standard Lenard chain generated by the recursion operator $K$ and by the privileged vector field $X$. This chain is composed by the vector fields

$$
X_{0}=X, \quad X_{1}=K X, \quad X_{2}=K^{2} X, \quad \cdots
$$

Let us assume that the first $n=\operatorname{dim} M$ vector fields of this chain are linearly independent, at least in some open region of $M$. Under the semisimplicity assumption

$$
X_{0} \wedge X_{1} \wedge \cdots \wedge X_{n-1} \neq 0,
$$

the Lenard chain defines a frame $\left\{X_{j}\right\}_{j=0, \ldots, n-1}$ and a coframe $\left\{\varepsilon^{j}\right\}_{j=0, \ldots, n-1}$ on $M$. By using the coframe $\varepsilon^{j}$ and the powers $K^{l}$ of the recursion operators, one may then define the third-order tensor field

$$
C=\varepsilon^{0} \otimes I d+\varepsilon^{1} \otimes K+\varepsilon^{2} \otimes K^{2}+\cdots+\varepsilon^{n-1} \otimes K^{n-1} .
$$

It defines a multiplicative structure on the tangent bundle. It can be shown that the tensor field $C$, and the privileged vector field $X$ obey the axioms of Hertling and Manin. Therefore each semisimple manifold of type $H_{1}$ is a semisimple F-manifold of Hertling and Manin. The converse is also true: any semisimple F-manifold is a semisimple manifold of type $H_{1}$, stripped of the privileged 1-form $\theta$. 


\section{Manifolds of type $H_{2}$ and beyond}

Having replaced the condition $\operatorname{Torsion}(K)=0$ by the weaker condition $\operatorname{Haantjes}(K)=0$, one looses an important property of bi-Hamiltonian manifolds. On these manifolds any short Lenard chain may be indefinitely prolonged into an infinite chain of closed 1-forms, to be referred to as the long Lenard chain. The process of extension is rather simple: it exploits the successive powers of the recursion operators. It is well-known, indeed, that on a bi-Hamiltonian manifold all the 1 -form $K^{j} \theta$ are closed. So, there is not end to a Lenard chain on a bi-Hamiltonian manifold.

This property fails on a manifold of type $H_{1}$. Normally it is not possible to prolong a short Lenard chain beyond its normal length by the iterated action of the recursion operator $K$. However, this difficulty may be circumvented. To construct short Lenard chains composed of more than three closed 1-form, the strategy is to replace the powers of the recursion operator by new recursion operators suitably selected. On imitation of the powers of $K$, the new recursion operators are assumed to commute. In its simplest form, this idea leads to the following definition of manifolds of type $\mathrm{H}_{2}$.

Definition 3. A manifold $M$ equipped with a privileged vector field $X$, a privileged 1-form $\theta$, and two recursion operators $K_{1}$ and $K_{2}$ is a manifold of type $H_{2}$ if:

1) Haantjes $\left(K_{j}\right)=0$;

2) $\left[K_{j}, K_{l}\right]=0$

3) $\mathrm{d}\left(K_{j} K_{l} \theta\right)=0$;

4) $\operatorname{Lie}_{X}\left(K_{j}\right)=0$;

5) $\operatorname{Lie}_{X}(\theta)=0$

for any value of the indices $j, l=0,1,2$. As usual, $K_{0}$ is the identity map on the tangent bundle $T M$.

The definition of manifold of type $H_{m}$ in presently completely obvious. Each time one adds a new recursion operator, and one insists that the above conditions be satisfied for any possible choice of the values of the indices $j, l$, ranging between 0 and $m$.

\section{WDVV equations}

Frobenius manifolds belong to the polygonal line defined by the vertices $H_{1}, H_{2}, \ldots, H_{\infty}$. Indeed, one may prove that a Frobenius manifold of dimension $n$ is a manifold of type $H_{n-1}$. Conversely, under very mild conditions of nondegeneracy, one may also prove that a manifold of type $H_{n-1}$ and dimension $n$ is a Frobenius manifold. The metric $g$ and the multiplication $C$ are reconstructed by using only the recursion operators $\left(K_{0}, K_{1}, K_{2}, \ldots, K_{n-1}\right)$, the privileged vector field $X$, and the privileged 1 -form $\theta$ of the manifold of type $H_{n-1}$. Thus the recursion operators have in the theory of Frobenius manifolds the same role they have in the theory of Poisson-Nijenhuis manifolds.

To prove these claims is outside the limits of the present note. For this reason, I limit myself to consider a concrete example. In this section I will study manifolds of type $\mathrm{H}_{2}$ and dimension 3 , and I will show that they are directly related to the WDVV equations, so important within the theory of Frobenius manifolds.

Let us start with the remark that a short Lenard chain on a manifold of type $\mathrm{H}_{2}$ is composed by six closed 1-forms. Locally I can consider these forms as exact, setting:

$$
\begin{array}{lll}
\theta=\mathrm{d} A, & K_{1} \theta=\mathrm{d} B, & K_{2} \theta=\mathrm{d} C, \\
K_{1}^{2} \theta=\mathrm{d} P, & K_{1} K_{2} \theta=\mathrm{d} Q, & K_{2}^{2} \theta=\mathrm{d} R .
\end{array}
$$


Let us assume that the first three 1-form $\mathrm{d} A, \mathrm{~d} B, \mathrm{~d} C$ are linearly independent. The corresponding potentials are, therefore, local coordinates on the manifolds $M$. Let us evaluate the action of the recursion operators $K_{1}$ and $K_{2}$ on this coordinate basis. One obtains

$$
\begin{array}{ll}
K_{1} \mathrm{~d} A=\mathrm{d} B, & K_{2} \mathrm{~d} A=\mathrm{d} C, \\
K_{1} \mathrm{~d} B=\mathrm{d} P, & K_{2} \mathrm{~d} B=\mathrm{d} Q, \\
K_{1} \mathrm{~d} C=\mathrm{d} Q, & K_{2} \mathrm{~d} C=\mathrm{d} R,
\end{array}
$$

where $P, Q, R$ are, so far, arbitrary functions of the coordinates $A, B, C$. These formulas define the most general pair of recursion operators generating a short Lenard chain of 1 -forms on a manifolds of type $\mathrm{H}_{2}$ and dimension 3 .

Let us now require that $K_{1}$ and $K_{2}$ commute. This condition restricts the choice of the arbitrary functions $P, Q, R$. The commutativity conditions are:

$$
K_{2} \mathrm{~d} P=K_{1} \mathrm{~d} Q, \quad K_{2} \mathrm{~d} Q=K_{1} \mathrm{~d} R .
$$

They imply that the functions $P, Q, R$ must solve the system of partial differential equations

$$
\begin{aligned}
& \frac{\partial P}{\partial A}=\frac{\partial P}{\partial C}\left(\frac{\partial Q}{\partial B}-\frac{\partial R}{\partial C}\right)+\frac{\partial Q}{\partial C}\left(\frac{\partial Q}{\partial C}-\frac{\partial P}{\partial B}\right), \\
& \frac{\partial Q}{\partial A}=\frac{\partial P}{\partial C} \frac{\partial R}{\partial B}-\frac{\partial Q}{\partial C} \frac{\partial Q}{\partial B}, \\
& \frac{\partial R}{\partial A}=\frac{\partial Q}{\partial B}\left(\frac{\partial Q}{\partial B}-\frac{\partial R}{\partial C}\right)+\frac{\partial R}{\partial B}\left(\frac{\partial Q}{\partial C}-\frac{\partial P}{\partial B}\right) .
\end{aligned}
$$

Another restriction comes from the existence of the vector field $X$. The symmetry conditions

$$
\operatorname{Lie}_{X}\left(K_{j}\right)=0, \quad \operatorname{Lie}_{X}(\theta)=0
$$

entail that the functions $A, B, C, P, Q, R$ have constant derivatives along $X$. In particular, this means that the vector field $X$ has constant components on the frame defined by the coordinates $A, B, C$. Thus

$$
X=a \frac{\partial}{\partial A}+b \frac{\partial}{\partial B}+c \frac{\partial}{\partial C}
$$

with $a, b, c$ constant parameters. Let us choose, for the sake of simplicity, $a=0, b=0, c=1$, so that

$$
X=\frac{\partial}{\partial C}
$$

The symmetry conditions then entail that the function $P, Q, R$ have the form

$$
P=\lambda C+\phi(A, B), \quad Q=\mu C+\psi(A, B), \quad R=\nu C+\chi(A, B),
$$

where $\lambda, \mu, \nu$ are arbitrary constants and the coordinate $C$ has been separated. Still for simplicity, let us choose $\lambda=1, \mu=0, \nu=0$. By inserting

$$
P=C+\phi(A, B), \quad Q=\psi(A, B), \quad R=\chi(A, B)
$$

into the above partial differential equations, one is left with

$$
\phi_{A}=\psi_{B}, \quad \psi_{A}=\chi_{B}, \quad \chi_{A}=\psi_{B}^{2}-\chi_{B} \phi_{B} .
$$


The solution is

$$
\phi=F_{A A}, \quad \psi=F_{A B}, \quad \chi=F_{B B}
$$

with

$$
F_{A A A}+F_{A A B} F_{B B B}-F_{A B B}^{2}=0 .
$$

This is one of the classical WDVV equations for Frobenius manifolds of dimension 3 [3]. This example shows that this WDVV equation comes out naturally from the study of short Lenard chains on manifolds of type $H_{2}$ and dimension 3. The general WDVV equations come analogously from the study of Lenard chain on manifolds of type $H_{n-1}$ and dimension $n$.

\section{Acknowledgements}

This note is a first account of a lasting research work done in collaboration with B. Konopelchenko. To him I address my warm acknowledgements for the permission to use part of the common work to prepare the conference and this note. Many thanks are also due to B. Dubrovin for the kind invitation to attend the conference on Geometrical Methods in Mathematical Physics.

\section{References}

[1] Hertling C., Manin Y., Weak Frobenius manifolds, Int. Math. Res. Not. 1999 (1999), no. 6, 277-286, math.QA/9810132.

[2] Hertling C., Frobenius manifolds and moduli spaces for singularities, Cambridge Tracts in Mathematics, Vol. 151, Cambridge University Press, Cambridge, 2002.

[3] Dubrovin B., Geometry of 2D topological field theories, in Integrable Systems and Quantum Groups (Montecatini Terme, 1993), Lecture Notes in Math., Vol. 1620, Springer, Berlin, 1996, 120-348, hep-th/9407018.

[4] Kosmann-Schwarzbach Y., Magri F., Poisson-Nijenhuis structures, Ann. Inst. H. Poincaré Phys. Théor. 53 (1990), 35-81. 